\title{
Descompactación biológica, una alternativa de solución a la compactación del suelo ${ }^{30}$
}

\section{Biological decompactation, an alternative of solution to the compacting of the soil}

\author{
Danny Ríos ${ }^{31}$; Arturo Estigarribia ${ }^{32}$ \\ DOI: https://doi.org/10.36003/Rev.investig.cient.tecnol.V2N2(2018)8
}

\section{RESUMEN}

La compactación del suelo, debido al colapso o disminución de los espacios de poros, es la causa más común de restricción física para el crecimiento y desarrollo de las raíces. Una vez que los poros han sido compactados hasta $0.2-0.3 \mathrm{~mm}$ de diámetro, es difícil que las raíces de los cultivos puedan penetrar libremente en el suelo. El objetivo de este trabajo es ofrecer una información actual sobre el método de descompactación biológica de los suelos, así como la aplicación práctica de los mismos. La utilización de especies con mayores potenciales de uso como son los abonos verdes que permitiría reducir a largo plazo la descompactación, así como desarrollar estrategias agronómicas que preserven el medio ambiente. El éxito de estos métodos biológicos depende de la selección de especies eficientes por tipo de suelo, su capacidad de descompactación y mejorador del suelo.

Palabras clave: Descompactación biológica; Abonos verdes; Compactación del suelo.

\footnotetext{
${ }^{30} \mathrm{Fecha}$ de recepción: septiembre 2018; fecha de aceptación: octubre 2018

${ }^{31}$ Estudiante de la Maestría en Ciencia del Suelo y Ordenamiento Territorial de la Facultad de Ciencias Agrarias de la Universidad Nacional de Asunción, San Lorenzo , Paraguay

${ }^{32}$ Egresado de la Facultad de Ciencias Agrarias de la Universidad Nacional de Asunción, San Lorenzo, Paraguay.
} 


\section{ABSTRACT}

Soil compaction, due to the collapse or reduction of pore spaces, is the most common cause of physical restriction for the growth and development of roots. Once the pores have been compacted to $0.2-0.3 \mathrm{~mm}$ in diameter, it is difficult for the roots of the crops to freely penetrate the soil. The objective of this work is to offer a current overview of the method of biological decompaction of soils, as well as the practical application of them. The use of species with greater potential for use such as green fertilizers that would reduce long-term decompaction, as well as develop agronomic strategies that preserve the environment. The success of these biological methods depends on the selection of efficient species by type of soil, their decompactability and soil improver.

Keywords: Biological decompaction; Green manure; Soil compaction. 


\section{INTRODUCCIÓN}

La realización de tareas como el paso de maquinaria y la aplicación de agroquímicos suele provocar el deterioro de las cualidades y funciones básicas del suelo. El suelo tiene componentes físicos, químicos y biológicos. Los físicos han sido ampliamente estudiados y, de cierto modo, solucionados con la introducción de una amplia gama de maquinaria agrícola destinada a diferentes labores como tractores, arados y subsoladores, entre otros (Sabaini \& Ávila 2013).

Considerando la importancia de realizar prácticas agrícolas que sean amigables con el medio ambiente surgen las prácticas biológicas y una de ellas sería la descompactación biológica que prácticamente consisten en el uso de abonos verdes, considerada una alternativa viable y económica para aportar nutrientes, carbono orgánico y mejorar las propiedades físicas de los suelos como por ejemplo la estructura de la misma. Está es una práctica agronómica importante que utiliza las plantas (especialmente leguminosas) como abono en rotación o sucesión y alternancia de cultivos; sus raíces incrementan el contenido de materia orgánica del suelo y ayudan a mejorar los problemas de compactación, considerando que las especies utilizadas poseen raíces pivotantes (García \& Martínez 2014). Este trabajo tiene como objetivo ofrecer una información actual sobre el método de descompactación biológica de los suelos, así como la aplicación práctica de los mismos.

\section{Desarrollo}

\section{La compactación del suelo}

La degradación de la estructura del suelo, muchas veces llamada compactación del suelo, es considerada la forma más seria de degradación de la tierra causada por las prácticas de labranza convencional. De todos los tipos de degradación de tierras, la degradación de la estructura del suelo es reversible y la misma puede ser mejorada mediante la aplicación de técnicas mecánicas que consisten en la utilización de subsoladores y la otro poca difundida, pero de gran importancia es la técnica que consiste en la utilización de especies biológicas (Brammer, 2000).

Es comúnmente considerada como el tipo de degradación de tierras más difícil de localizar y reconvertir, siendo la razón que la degradación de la estructura del suelo un fenómeno subsuperficial, que a diferencia de la erosión y salinización requiere análisis físicos antes de ser revelada e identificada su extensión, naturaleza, y causa. La combinación del alto costo y la invisibilidad hacen de la degradación de la estructura del suelo un gran riesgo para el desafío de la seguridad alimentaria global (Yadav et al., 2017)

La compactación de suelos es causada por las prácticas agrícolas convencionales, es reversible y su ocurrencia es previsible o, al menos, controlable. Es un problema de carácter mundial que abarca todos los suelos y niveles de producción agrícola. Es el tipo más difícil de degradación de suelos para localizar y racionalizar debido a que es un fenómeno que ocurre sobre todo debajo de la superficie como lo mencionan autores de párrafos anteriores (Food and Agriculture Organization, 2015).

La compactación del suelo produce un aumento en su densidad aparente, aumenta su resistencia mecánica, destruye y debilita su estructuración. Todo esto hace disminuir la porosidad total y la macroporosidad (porosidad de aireación) del suelo. Los efectos que la compactación produce, se traducen en un menor desarrollo del sistema radical de las plantas y, por lo tanto, un menor desarrollo de la planta en su conjunto, lo que redunda en una menor producción (Gonzalez et al., 2009; Müller 
et al., 2001).

La compactación es uno de los principales procesos de degradación de los suelos agrícolas (Horn et al., 2003) y es expresada por el aumento de la densidad del suelo y por la reducción de su espacio poroso en respuesta a un histórico de cargas o presiones ejercidas en la superficie, especialmente en elevada humedad (Baver et al. 1972 citado por Silveira et al., 2008).

\section{Mecanismos de descompactación}

La metodología usual para romper este tipo de compactación es mediante la utilización de implementos mecánicos tipo subsoladores. También arados de cincel o rastra de discos cuando la compactación es de tipo más superficial y lógicamente de acuerdo al sistema de trabajo, en siembra directa o convencional. Otra alternativa, no difundida en nuestro medio y tampoco estudiada en profundidad, es el aprovechamiento de las raíces pivotantes de determinadas especies vegetales para penetrar las capas compactadas. Estas raíces, una vez descompuestas, dejan en el suelo canales por donde pueden circular el agua, el aire y penetran las raíces de los cultivos posteriores. Las especies de la familia Crucíferas resultan muy interesantes a estos efectos. La bibliografía menciona que los poros creados por este método biológico tendrían mayor estabilidad que los creados por medios mecánicos, posiblemente debido a la presencia de materia orgánica que los recubre, producto de la descomposición de las raíces (FAO, sf).

Según Dexter (1991) citado por Calonego et al. (2011), los bioporos derivado de raíces y lombrices de tierra hacen parte de procesos naturales de mejoría de las características físicas del suelo razón por la cual es importante el estudio de la descompactación biológica para garantizar mejoras en la estructura del suelo que se traduzcan en una mayor producción de manera sostenible.
Los abonos verdes juegan un papel importante en la restauración de tierras degradadas, sus efectos más importantes serían incremento de la materia orgánica y ciclo nutricional, fijación de nitrógeno, control de malezas, conservación del suelo, mejoramiento de la humedad del suelo, labranza cero, control de enfermedades de plantas y nematodos Los abonos verdes y los cultivos de cobertura pueden desempeñar un papel importante en la restauración de las tierras degradadas. Su uso puede resultar en un incremento tan significativo de la fertilidad del suelo que es posible hablar no sólo de su conservación, sino también de restauración y recuperación del suelo el cual se resalta el efecto de las raíces para el movimiento natural del suelo colaborando para la recuperación física del mismo (Brunch, 2004).

Según SAGARPA (2009) los abonos verdes son los principales descompactadores o escarificadores biológicos, se caracterizan por ser de rápido crecimiento y de follaje denso cuya función no es el consumo humano o pecuario sino la incorporación en el suelo para mejorar sus propiedades con fines agrícolas. Preferentemente en estado de floración, se entierran en el suelo para mejorar la fertilidad, el contenido de carbono orgánico, las propiedades físico-químicas de los suelos, aumentar los contenidos de materia orgánica, la capacidad de retención de humedad y el deterioro de la estructura del suelo, entre otros, como efectos desfavorables. Los cultivos al no cubrir sus demandas nutricionales, por el uso exclusivo de abonos verdes, requieren la aplicación complementaria de fertilizantes químicos.

Alleoni (1997) citado por Calonego et al. (2011), mencionan que con el uso de plantas descompactadoras se pueden proporcionar un rompimiento más uniforme de la camada compactada, más allá de contribuir en la mejora de los agregados del 
suelo. Araujo et al. (2004), sostienen que los manejos mecánicos de la compactación del suelo son de corta duración a diferencia de la compactación biológica y más aún si no son consideradas medidas preventivas como la utilización de plantas con sistema radicular abundante y que incremente el contenido de materia orgánica de manera a estabilizar los agregados del suelo. Conforme a lo mencionado por Cardoso et al. (2003), para evitar o minimizar la compactación, la disminución de las operaciones agrícolas no es la solución, sino que deben ser consideradas las rotaciones de cultivos con especies que produzcan gran cantidad de masa para la cobertura del suelo y que, principalmente, posean un sistema radicular profundo y con gran volumen, capaz de producir canales disminuyendo de esa manera la compactación lo que es conocido hoy en día como descompactación biológica.

En la agricultura mecanizada, los pies de arado se forman generalmente a 12-30 $\mathrm{cm}$ de profundidad, dependiendo del implemento empleado y de su profundidad normal de trabajo. Sin embargo, las capas densas formadas naturalmente pueden ocurrir a cualquier profundidad, por lo que para implementar las plantas descompactadoras uno debe primeramente realizar un diagnóstico de manera a considerar la profundidad en la que se encuentra el problema y a partir de eso poder utilizar la planta descompactadora con las características adecuadas que permitan la solución del problema (Brunch, 2004).

Gançalves et al. (2006), mencionan que la presencia de una camada compactada influyen en el crecimiento de las raíces, por lo que las especies que no poseen una raíz pivotante y buena densidad de longitud radicular acumulan gran parte de su crecimiento en la parte superficial, a diferencia de lo que ocurre con especies que presentan características mencionadas anteriormente, como por ejemplo Mileto
(Pennisetum glaucum) que es un especie capaz de emitir raíces y explorar el suelo, lo que resulta en beneficios a la planta y al suelo.

Según la DINCAP/MAG (2008), la descompactación biológica consiste en la utilización de plantas rústicas, con sistema radicular pivotante y buena capacidad de penetración en el suelo, que promuevan la descompactación de las camadas adensadas. Sirven para tal efecto especies como el kumanda yvyra'i (Cajanus cajans), nabo forrajero pivotante (Rhaphanus sativus), lupino blanco (Lupinus albus), Avena negra (Avena strigosa), entre otras. Para $\mathrm{Cu}-$ billa et al. (2002), la eficiencia del método biológico depende del estado inicial de compactación del suelo. En el cual el comportamiento de las raíces de las especies a ser utilizadas como descompactadoras biológicas juegan un rol importante considerando que la misma debe de ser una raíz pivotante con buen desarrollo en suelos donde se registra una camada compactada.

\section{Características de especies utilizadas como descompactadores biológicos en el Paraguay}

El Lupino Blanco (Lupinus albus) es una leguminosa anual herbácea erecta de porte medio, buena fijadora de nitrógeno a través de las bacterias que forman nódulos en sus raíces aportando alrededor de $90 \mathrm{Kg}$.ha-1 de nitrógeno. Su sistema radicular es bastante desarrollado (raíz pivotante), pudiendo alcanzar 2 metros o más de profundidad, con buen efecto sobre las características físicas, químicas y biológicas del suelo. Soporta temperaturas de $3-4{ }^{\circ} \mathrm{C}$. En la fase inicial de crecimiento es sensible a la sequía. Con excesos de humedad sufre ataque de antracnosis que puede matar el cultivo. Este problema se reduce con rotaciones y mezclando abonos verdes como con la avena negra. Produce 4 ton.ha-1 de materia seca. Cuando 
se siembra en forma densa por lo menos con $80 \mathrm{~kg}$./semilla /ha. Es indicado para cultivos con altas exigencias de Nitrógeno. Siembras de otoño. Si se siembra a más de $4 \mathrm{~cm}$. de profundidad se dificulta la emergencia. Se recomienda asociarlo con avena negra (Florentin et al., 2001).

El Kumanda yvyra'i (Cajanus cajans), es una planta rústica que se caracteriza por su gran aporte de proteínas. Debido a su gran capacidad de fijar nitrógeno del aire en el suelo y producir buena cantidad de biomasa, con lo cual provee materia orgánica a través de sus ramas leñosas de lenta descomposición, es recomendable, principalmente, para la recuperación de suelos degradados. Sus raíces son profundas, actúan como subsolador del suelo y pueden llegar hasta más de cinco metros de profundidad, rompiendo las capas duras (pie de arado), que en la mayoría de las pequeñas fincas se encuentran a 15 o $20 \mathrm{~cm}$. de profundidad (Caballero, 2007)

El nabo forrajero (Rhapanus sativus), es una planta anual que pertenece a las crucíferas, herbácea erecta de porte medio. Crecimiento inicial rápido, no es muy rústico y produce alta cantidad de biomasa en condiciones de mediana o alta fertilidad hasta 5 ton.ha-1 de materia seca. En suelos degradados no se recomienda su uso por bajo rendimiento. Con raíces pivotantes profundas es capaz de romper camadas compactas de suelo, reciclar nutrientes en especial N2 y solubiliza elementos como fósforo que quedan disponibles para el cultivo siguiente. Es muy eficiente en la supresión de malezas por el rápido sombreado que realiza del suelo y su efecto alelopático. La rápida degradación de su biomasa luego de muerto permite un rápido crecimiento de malezas y la ocurrencia de problemas asociados con baja cobertura. Esto se puede solucionar con la asociación con otros abonos verdes como avena negra. Se siembra al voleo $20 \mathrm{~kg}$.ha-1 de semilla, en siembras asociadas se usa menos (Florentin et al., 2001).

Uno de los aspectos importantes por la cual son utilizadas las crucíferas en sistemas conservacionistas es la capacidad que tienen algunos tipos en desarrollar raíces engrosadas que al momento de descomponerse dejan grandes canales que mejoran la estructura y drenaje del suelo. Además, son importantes recicladores de nutrientes debido a su sistema radicular (Gilsanz, 2008).

La avena negra (Avena strigosa) es una gramínea anual, erecta, de porte medio, muy bien adaptada a las condiciones de clima y suelo de la Región Oriental del Paraguay. Produce 4 a 5 toneladas por hectáreas de materia seca, en suelos de mediana y alta fertilidad; sin embargo, desarrolla muy poca biomasa en suelos muy degradados, entre 1 a 3 toneladas de materia seca por hectárea. Esta planta de cobertura responde notoriamente a la fertilización química, principalmente nitrogenada. En suelos pobres, aun cuando produce poca biomasa, controla bien las malezas, por medio del efecto alelopático y mejoran la estructura del suelo a través de la densidad de las raíces que tienden a descompactar el pie de arado que se encuentran a los 10-15 $\mathrm{cm}$ de profundidad (DINCAP/MAG 2008).

Los suelos en SD, mediante el efecto de las raíces y de la actividad biológica, asociado a procesos de humectación y desecamiento, tienen la capacidad de recuperar la estructura. Este proceso, que depende de las características de cada lote (cobertura, materia orgánica) puede demorar varios años (Gerstert y Bacigaluppo, 2004).

\section{Estudios de la aplicación de abonos verdes como descompactadoras bioló- gicos}

En trabajos realizados con la utilización de las especies mencionadas denominadas comúnmente como abonos verdes se destacan ciertas especies como descompacta- 
dores biológicos del suelo; Silveira et al. (2008),en su trabajo de comparación del método mecánico vs método biológico de descompactación pudo constatar que la escarificación biológica es eficiente en el aumento de la macroporosidad del suelo , disminución de la resistencia a la penetración y mejora en la filtración del agua en un suelo inicialmente compactado, además es importante destacar que las mejoras físicas obtenidas con la misma son más persistentes en relación a la escarificación mecánica. La asociación de nabo forrajero con avena negra aumenta la producción de biomasa del suelo, lo cual indica que dicha asociación es efectiva para la mejora de la calidad del suelo el cual fue reflejado en un buen rinde en el cultivo de soja.

Según Kubota et al. (2005), en evaluaciones realizadas después del manejo de una parcela donde fue utilizado nabo forrajero como cultivo de cobertura, no observaron un efecto positivo inmediato en las condiciones físicas de un Oxisol de textura media, donde por el contrario observaron un aumento en la densidad del suelo en la camada de 0 - $0.05 \mathrm{~m}$. Este efecto dichos autores lo atribuyeron a la presión ejercida en suelo por las raíces del nabo forrajero, debido al gran diámetro de su raíz pivotante principal. Sin embargo, en evaluaciones posteriores realizadas se observaron un aumento en las macroporosidades, porosidad total, y estabilidad de los agregados del suelo.

Calonego et al. (2011), sostienen que la densidad es un factor importante a considerar para utilizar al nabo forrajero como descompactador biológico considerando que la densidad recomendada para cobertura es de 15 a $20 \mathrm{~kg}$. ha-1 de semillas, lo cual debe de aumentarse de manera a disminuir el diámetro y aumentar el número de raíces pivotantes por unidad de área, para conseguir una mejora en la estructuración del suelo de manera más uniforme.
Según la Unión de Coperativas (2015), considerando sus experiencias, el monocultivo, la ausencia de rotación de cultivos adecuados con cultivos de cobertura, como también el manejo inadecuado de plagas, enfermedades y malezas obligan al productor a una mayor repetición de diferentes aplicaciones. Esto significa más frecuencia de tránsito con las máquinas en los terrenos, lo que ocasiona la compactación del suelo, por lo que la introducción de especies con raíces pivotantes en un sistema de producción es crucial para mantener una producción sustentable, mediante una mejora en las propiedades físicas y químicas.

Según Calonego et al. (2011), en un trabajo denominado desenvolvimiento de plantas de cobertura en suelo compactado pudieron observar que la especie Brachiaria ruziziensis obtuvo un buen crecimiento radicular en la camada intermedia, a diferencia del sorgo que obtuvieron una mayor acumulación de raíces en camadas más profundas lo cual demuestra que dicha especie posee un buen comportamiento para actuar como descompactador biológico.

Longaretti et al. (2017), mediante un estudio de plantas descompactadoras y su efecto sobre las propiedades físicas del suelo, pudieron concluir que el empleo de avena blanca, reduce la resistencia del suelo en la camada de $0.1-0.2 \mathrm{~m}$, la cual se ve favorecido por el contenido de humedad en el suelo el cual facilita la penetración de las raíces a dichas profundidades y garantizan la utilización de dicha especie como una alternativa a la solución del problema de compactación a las profundidades mencionadas bajo los factores en que fue manejado el experimento.

Según Abreu et al (2004), la eficacia de la ruptura de la camada compactada del suelo depende de la propiedad hídrica o mecánica del suelo utilizada como indicadora. Así utilizando el indicador conductividad hidráulica de un suelo saturado, la 
escarificación biológica que consistió en cultivo mínimo de Crotalaria con siembra de Crotalaria spectabilis con densidad aproximada de $60 \mathrm{~kg}$. ha-1 de semillas, fue más eficaz a medio plazo, en la ruptura de la capa compactada y establecimiento de poros conductores de agua del que la escarificación mecánica del suelo. En contrapartida, si el indicador es la resistencia a la penetración y el interés es la reducción de la resistencia a las raíces, el resultado obtenido fue inverso.

En un estudio de desenvolvimiento de plantas descompactadoras sometidos a diferentes niveles de compactación Zancan et al. (2017), pudieran observar que el consorcio de nabo forrajero y vicia presentaron un buen desarrollo de sistema radicular en comparación con la de avena blanca en un suelo compactado bajo diferentes niveles de tráfico.

Pereira et al. (2015), en un trabajo de in- vestigación en el cual probaron la influencia de la densidad del suelo en los atributos de la parte aérea y sistema radicular pudieron observar que la eficacia de la ruptura de la camada compactada del suelo depende de la propiedad hídrica o mecánica del suelo utilizada como indicadora.

Abreu et al. (2004) en su trabajo denominado escarificación biológica y mecánica para la reducción de la compactación en un Ultisol de textura franco arenosa sobre plantío directo pudieron notar que la resistencia mecánica a la penetración fue máxima en la camada $0.075 \mathrm{~m}$ en tratamientos sin preparación de suelo, en cambio en un suelo escarificado la resistencia a la penetración ocurrió a mayor profundidad (0.175 m).

\section{CONCLUSIONES}

Considerando las revisiones bibliográficas consultadas podemos concluir que para que la descompactación biológica sea efectiva, la misma se debe aplicar en suelos no muy compactados, es decir con suelos con resistencia a la penetración inferior a 2.0 PSI. Mediante el aprovechamiento de las raíces pivotantes de determinadas especies vegetales para penetrar las capas compactadas. Estas raíces, una vez descompuestas, dejan en el suelo canales por donde pueden circular el agua, el aire y penetran las raíces de los cultivos posteriores.

Los abonos verdes juegan un papel importante en la restauración de tierras degradadas, sus efectos más importantes serían incremento de la materia orgánica y ciclo nutricional, fijación de nitrógeno, control de malezas, conservación del suelo, mejoramiento de la humedad del suelo, labranza cero, control de enfermedades de plantas y nematodos.

El manejo mecánico de la compactación del suelo es de corta duración a diferencia de la compactación biológica y más aún si no son consideradas medidas preventivas como la utilización de plantas con sistema radicular abundante y que incremente el contenido de materia orgánica de manera a estabilizar los agregados del suelo.

Conforme a lo mencionado, para minimizar la compactación, la disminución de las operaciones agrícolas no es la solución, sino que deben ser consideradas las rotaciones de cultivos con especies que produzcan gran cantidad de masa para la cobertura del suelo y que, principalmente, posean un sistema radicular profundo $\mathrm{y}$ con gran volumen, capaz de producir canales disminuyendo de esa manera la compactación lo que es conocido como compactación biológica. 
La descompactación biológica es una alternativa válida para enfrentar los problemas de compactación de suelos, considerando que dicha práctica proporciona una mejora en la estructura del suelo de manera más estable a diferencia de lo que proporciona la descompactación mecánica que otorga una solución por tiempo limitado.

\section{BIBLIOGRAFÍA}

1. Abreu, S.; Reichert, J.\& Reinert, D. (2004). Escarificação mecânica e biológica para a redução da compactação em argissolo franco-arenoso sob plantio direto. R. Bras.Ci. Solo, 32,1723-1734.

2. Araujo, M.; Tormena, C.; Inoue, T.\& Costa, A. (2004). Efectos de escarificación en la calidad física de un Latossolo Vermelho distroférrico después de trece años de siembra directa. Revista Brasileira de Ciencia del Suelo,Vicosa. 28 (3),495504.

3. Brammer, H. (2000). Ploughmans and tillage problems. In: Agroecological aspects of agricultural research in Bangladesh.Dhaka, 151-158.

4. Brunch, R. (abril de 2004). Adopción de abonos verdes y cultivos de coberturas. Recuperado el 14 mayo 2018, de Revista de Agroecología: http://leisa-al.org/ web/images/stories/revistapdf/vol19n4. pdf\#page $=10$

5. Calonego, J.; Gómez, T.; Dos Santos, H. \& Tiritan, C. (2011). Desenvolvimiento de plantas de cobertura en suelo compactado. Bioscience Journal,Uberlandia, 27 (2), 289-296.

6. Cardoso, E.; Zotarelli, L.; Piccinin, J.\& Torres, J. (2003). Distribuição do sistema radicular da cultura da soja em função do manejo do solo. Congresso Brasileiro de Ciencia del Suelo. Ribeirão Preto. Anais. Ribeirão Preto: Sociedade Brasileira de Ciência do Solo.

7. Cubilla, M.; Reinert, D.J.; Aita,
C.\& Reichert, J.M. (2002). Plantas de cobertura do solo: uma alternativa para aliviar a compactação em sistema plantio direto. R. Plantio Direto, 71, 29-32.

8. DINCAP/MAG (Dirección $\mathrm{Na}$ cional de Coordinación y Administración de Proyectos/ Ministerio de Agricultura y Ganadería, Paraguay). (2008). Recuperado el 02 junio de 2018, de El subsolado del suelo: http://www.stp.gov.py/cooperacion/ giz/wp-content/uploads/2015/05/El-subsolado.pdf

9. FAO (Food and Agriculture Organization). (2015). Recuperado el 15 de mayo de 2018, de Los suelos están en peligro, pero la degradación puede revertirse: http:/www.fao.org/news/story/es/ item/357165/

10. FAO (Food and Agriculture Organization).Sf. Recuperado 14 de mayo de 2018, de Conservación de los recursos naturales para una agricultura sostenible. Soluciones para la compactación del suelo: http://www.fao.org/ag/ca/Training_Materials/CD27Spanish/sc/soil_compaction. pdf

11. Florentín, M; Peñalva, M.; Calegari,A.\& Derpsch, R. (2001). Abonos verdes y rotación de cultivos en siembra directa: pequeñas propiedades. San Lorenzo, Py. MAG-GTZ (Proyecto de Conservación de suelos MAG-GTZ).

12. Gançalves, W.; Jiménez, R.; De Araújo, J.; De Assis, R.; Silva, G.\& Pires, F. (2006). Sistema radicu- 
lar de plantas de cobertura sob compactação do solo. Revista Ingeniería Agrícola, Jaboticabal, 26 (1), 67-75.

13. García, S; Martínez, M. (2014). Recuperado el 15 de mayo de 2018, de Abonos verdes:http://www.sagarpa.gob. $\mathrm{mx} / \mathrm{des}$ arrolloRural/Documents/fichasCOUSSA/Abonos\%20Verdes.pdf

14. Gerstert, G. \& Bacigaluppo, S. (2004). Consecuencias de la densificación por tránsito en argiudoles del sur de Santa Fe. In: resúmenes del XIX congreso argentino de la ciencia del suelo.

15. Gilsanz, J. (2008). Recuperado el 11 de junio de 2018, de Jornada de abonos verdes: Jardín de abonos verdes de invierno. INIA (Instituto Nacional de Investigación Agropecuaria):http://www.inia.uy/ Publicaciones/Documentos\%20compart idos/18429261108095122.pdf

16. González, O; Iglesias, C \& Herrera, M. (2009). Recuperado el 15 de mayo de 2018, de Análisis de los factores que provocan compactación del suelo agrícola: http://www.redalyc.org/ html/932/93215937011/

17. Horn, R.; Way, T.\& Rostek, J. (2003). Effect of repeated tractor wheeling on stress/strain properties and consequences on physical properties in structured arable soils. Soil Till.Res., 73, 101-106.

18. Kubota, A.; Hoshiba, K. \& Bordon, J. (2005). Green manure turnip for soybean based no-tillage farming systems in eastern Paraguay. Sci. Agric., 62,150158.

19. Longeratti, M.; Zancan, A.; Dos Santos, N.; Alves, A.\& Pérez, D. (2017). Plantas Descompactadoras e seu efeito sobre as propriedades físicas de um nitossolo vermelho. Maceio, Br. In: Resúmenes del XLVI Congreso Brasileiro de Enghenaria
Agrícola-CONBEA.

20. Müller, M.; Ceccon, G.\& Rosolem, C. (2001). Influência da compactação do solo em subsuperfície sobre o crescimento aéreo e radicular de plantas de adubação verde de inverno. Revista Brasileira de Ciência do Solo, Viçosa., 25(3), 531-538.

21. Pereira, L.; Dalla, S.; Bonfim, B.; Damacena, E.\& Da Silva, F. (2015). Influência da densidade do solo em atributos da parte aérea e sistema radicular de crotalaria. Pesq. Agropec. Trop. Goiânia, 45(4),.464-472.

22. Sabaini C. \& Ávila G. (2015). Manual de determinación de la condición biológica de suelo in situ e in visu en los sistemas agrícolas. Programa de Restauración Biológica de Suelo (RBS). Centro Regional de Innovación Hortofrutícola de Valparaíso.Quillota. Chile. 55 p.

23. SAGARPA (Secretaría de Agricultura, ganadería, desarrollo rural, pesca y alimentación). (2009). Recuperado el 03 de junio de 2018, de Prácticas vegetativas y agronómicas complementarias al proyecto integral: http://www.sagarpa.gob. $\mathrm{mx} /$ desarrolloRural/noticias/2012/Julio/ Documents/Pr\%C3\%A1cticas\%20vegetativas $\% 20 \mathrm{y} \% 20$ agron $\% \mathrm{C} 3 \% \mathrm{~B} 3$ micas.pdf

24. Silveira, R.; Carneiro, J.; Schneider, S.; Lanzanova, M.; Cauduro, V.\& Bragagnolo, J. (2008). Eficiência da escarificação mecânica e biológica na melhoria dos atributos físicos de um latossolo muito argiloso e no incremento do rendimento de soja. Revista Brasileira de Ciencias del Suelo, 32 (1), 1723-1734.

25. UNICOOP (Unión Central de Cooperativas). (2015). Recuperado el 15 de junio de 2018, de Prácticas recomendadas para el buen manejo de suelos en la producción de granos. Santa Rita, Py: 
http://www.unicoop.com.py/admin/archivos/manual-de-buen-manejo-de-suelosen-la-produccion-de-granos.pdf

26. Yadav, M.R.; Parihar, R.K.; Yadav, S.L.; Jat, A.K.; Singh, H.; Ram, R.K.; Meena, M.; Singh, V.K. \& Meena, N. (2017). Conservation Agriculture and Soil Quality.An Overview. Int.J.Curr.Microbiol.App.Sci, 6(2),1-28.

27. Zancan, A.; Longeratti, M.; Verardi, J.; Conte, P.\& Da Rosa, D. (2017). Desenvolvimento de plantas descompactadoras submetidas a diferentes níveis de compactação. Maceio, Br. In: Resúmenes del XLVI Congreso Brasileiro de Enghenaria Agrícola-CONBEA. 